\title{
Resource Sharing via Planed Relay for HWN*
}

\author{
Chong Shen, Susan Rea, and Dirk Pesch \\ Centre for Adaptive Wireless Systems, Department of Electronic Engineering, Cork Institute of Technology, Ireland \\ Correspondence should be addressed to Chong Shen, chong.shen@cit.ie
}

Received 23 October 2007; Revised 22 February 2008; Accepted 1 April 2008

Recommended by J. Wang

\begin{abstract}
We present an improved version of adaptive distributed cross-layer routing algorithm (ADCR) for hybrid wireless network with dedicated relay stations $\left(\mathrm{HWN}^{*}\right)$ in this paper. A mobile terminal (MT) may borrow radio resources that are available thousands mile away via secure multihop RNs, where RNs are placed at pre-engineered locations in the network. In rural places such as mountain areas, an MT may also communicate with the core network, when intermediate MTs act as relay node with mobility. To address cross-layer network layers routing issues, the cascaded ADCR establishes routing paths across MTs, RNs, and cellular base stations (BSs) and provides appropriate quality of service (QoS). We verify the routing performance benefits of $\mathrm{HWN}^{*}$ over other networks by intensive simulation.
\end{abstract}

Copyright (C) 2008 Chong Shen et al. This is an open access article distributed under the Creative Commons Attribution License, which permits unrestricted use, distribution, and reproduction in any medium, provided the original work is properly cited.

\section{INTRODUCTION}

Time Division Multiple Access (TDMA)-based digital cellular standard global system for mobile (GSM) was first deployed in 1990 with a new $900-\mathrm{MHz}$ band. However, due to uneven nature of the time-varying spatial distribution [1], network performance metrics are not sufficient for today's wireless network where more ad hoc features are being introduced.

To effectively manage problems stated above, we propose to combine the advantages of different networks so that the Mobile Terminal (MT) can utilise an optimised MANET, the base-station-oriented network (BSON) and the relay services. Figure 1 presents hybrid wireless network with relay nodes $\left(\mathrm{HWN}^{*}\right)$, the relay nodes $(\mathrm{RNs})$ of core network compose a mesh-like structure connected to the internet protocol (IP) networks through RN gateways, while base stations (BSs) are connected to the IP networks via switches. In rural places without infrastructure support as indicated in Figure 1, two MTs may communicate directly, or through intermediate MTs. When an MT transmits packets to a BS through RNs, the RNs extend the signalling coverage of BSON thus we can expect an enhanced resource-sharing performance.

An adaptive distributed cross-layer routing (ADCR) algorithm is proposed for $\mathrm{HWN}^{*}$ based on [2] using the minimal number of hops and considering routing model dynamic switching to reduce latency, preserve communications, deliver good overall throughput/per node throughput, and extend the $\mathrm{HWN}^{*}$ coverage. A cross-layer network design [3] that seeks to enhance the system performance by jointly designing MAC and NETWORK layers is adopted. We analyse in design stage the theoretical cellular network media access capacity, multihop traffic relaying issues, and inter network traffic handovers [4]. The cascaded ADCR then includes three subpacket transmission modes labeled as one-hop ad-hoc transmission (OHAHT) for point-topoint ad hoc direct communication, multihop combined transmission (MHCT) for radio resource relaying using fixed RNs or MTs, and cellular transmission (CT) for traditional cellular service. In rural places without infrastructure RN support, the MHCT transmission mode can be implemented on selforganised ad hoc nodes for supporting multihop communication as long as: (i) The resource of relaying MTs is contention-free, (ii) the migration range of relaying MTs is limited, and (iii) the speed changes of relaying MTs in sampling times have limited influence on routing.

The paper begins with a heterogenous wireless networks $\mathrm{RN}$ incorporation discussion, including the comparison work between proposed $\mathrm{HWN}^{*}$ framework stage I and $\mathrm{HWN}^{*}$ framework stage II. We present two pre-engineered RNs positioning algorithms in Section 3. In Section 4, we 


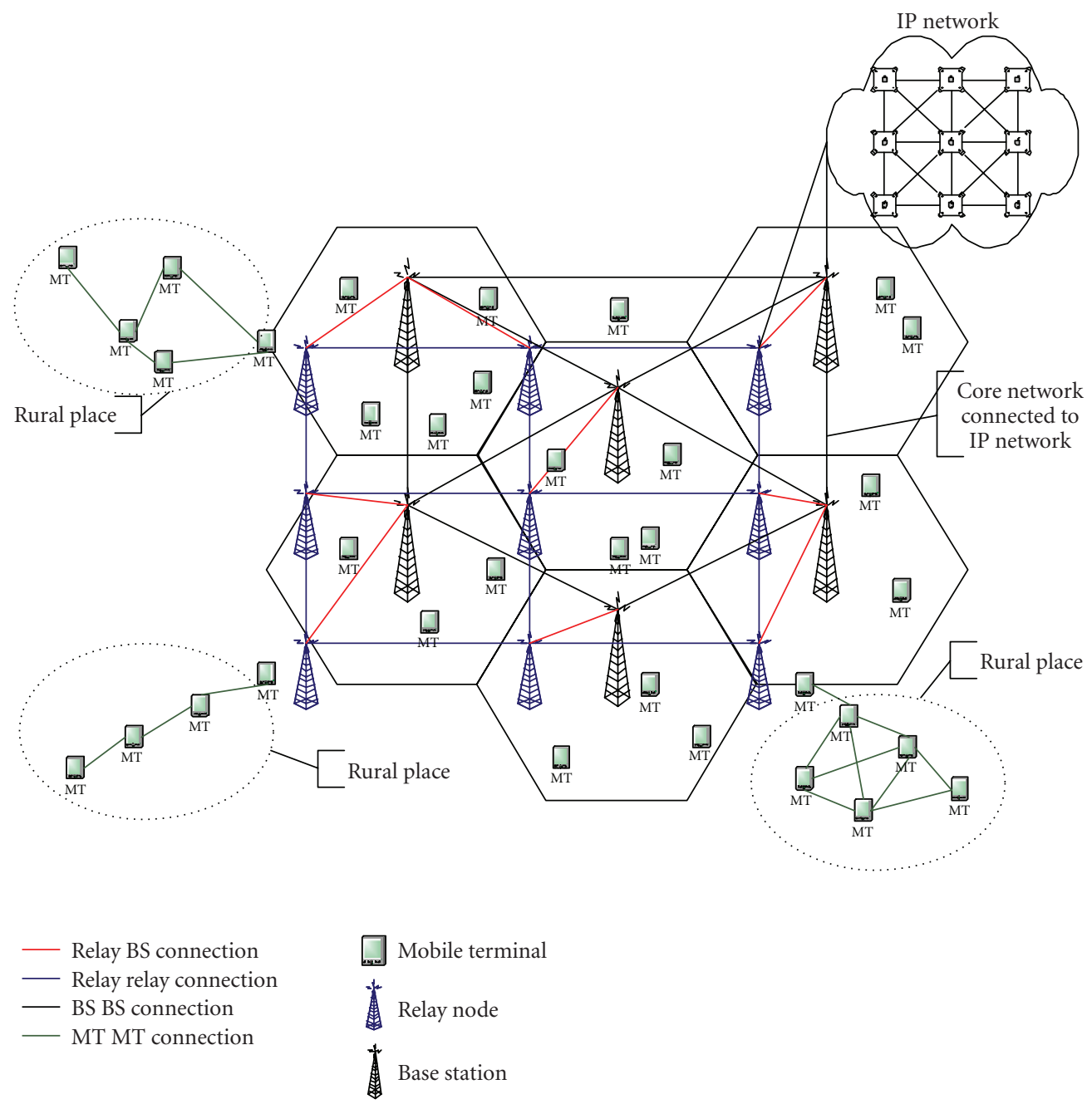

FIGURE 1: The hybrid wireless network with fixed relay stations.

discuss three traffic transmission modes with emphasis on MHCT mode in copy with newly included packet relaying environment. The ADCR performance of the $\mathrm{HWN}^{*}$ under various scenarios is evaluated in Section 5 to address network capacity, per MT throughput, access speed, and end-to-end delay. Finally in Section 6, conclusions are made with future research outlook.

\section{HETEROGENOUS WIRELESS NETWORKS}

The further balance of radio resource in heterogeneous networks or hybrid wireless networks requires assistant equipment functioned-like internetwork switcher, thus we introduced a new node structure (RN), and further divided it into heterogeneous $R N$ that uses different radio access technology (RAT) with common or different sets of transmission resources for its links and homogeneous relay node that uses the same radio access technology and mode in a common set of transmission resources for its entire links. For example, the IST-WINNER [5] project proposes to share the same RAT with BSs, RNs, and MTs to realise a dynamic spectrum usage.
Multiple noninterfering relay frequencies operate in parallel through the use of intelligent radios. The spectrum where an $\mathrm{RN}$ operates can be leased for a limited time depending on network status. The spectrum on which it is operating is reclaimed when network performance improves. Two RNs operating on noninterfering spectrums form a network relay link with multiple orthogonal bands. Multiple nodes within range of each other may also transmit simultaneously on different channels without relying on a media access protocol or distributed scheduling algorithm to resolve contention.

Focus on different design objectives, the iCAR [6] is derived from existing cellular networks and enables the network to achieve theoretical capacity through adaptive traffic load balancing. The SOPRANO [7] is a scalable architecture that assumes the use of asynchronous codedivision multiple access (CDMA) with spreading codes to support high-data-rate internet and multimedia traffic. It is similar to iCAR other than IP network support and cross network connection methods. We summarise in Table 1 the main research improvements from the HWN* stage I [4] to the $\mathrm{HWN}^{*}$ stage II. The comparison between the 
TABLE 1: Research improvement of $\mathrm{HWN}^{*}$ framework stage II.

\begin{tabular}{|c|c|c|}
\hline project & HWN* framework stage I & $\mathrm{HWN}^{*}$ framework stage II \\
\hline Main objectives & $\begin{array}{l}\text { Incorporate a MANET to increase system capacity } \\
\text { while realising differentiated QoS services }\end{array}$ & $\begin{array}{l}\text { Stage I + Investigation on places without infrastruc- } \\
\text { ture support }\end{array}$ \\
\hline Basic infrastructure & BSON, BSON with RN, MANET with RN & $\begin{array}{l}\text { BSON, BSON with RN, MANET with RN and } \\
\text { MANET }\end{array}$ \\
\hline Routing issues & BS switch and RN assisted traffic diversion & $\begin{array}{l}\text { Cascaded and distributed routing with three trans- } \\
\text { mission modes }\end{array}$ \\
\hline Mode movement issues & Attractor points model & $\begin{array}{l}\text { Costudy of user movement model and RN placement } \\
\text { algorithm }\end{array}$ \\
\hline Congestion control & QoS-based session congestion control algorithm & QoS-based session congestion control algorithm \\
\hline RN positioning scheme & Fix point $\mathrm{RN}$ positioning & RN positioning considering node movement pattern \\
\hline Load balance & QoS-based multihop load balancing & QoS-based multihop load balancing \\
\hline Call admission & The BS coordinated admission & Distributed session admission algorithm \\
\hline
\end{tabular}

iCar, multipower architecture for cellular network (MuPAC), hybrid wireless network (HWN) without RN support, WINNER, SOPRANO, and MCN can be found in $[4,8]$ with the identification of technologies used.

Consider a cellular handover scenario in Figure 2 where MT A is currently connected to MT B and is moving out of Cell 1 into Cell 6. A request for a BS handover will be sent as soon as the power level by MT A goes below a certain threshold (trajectory indicated by red dotted line). A successful handover will take place within a few hundred milliseconds depending on speed before the received power from BSs reaches an unacceptable level. When MT A arrives in Cell 6 , if the congestion persists in cell 6 for a period of time during which the MT moves farther away from the other neighbouring cell border, thus causing the received power level from $\mathrm{BS}$ A to fall below the acceptable level, handover will fail and the call will be permanently terminated.

However, in MHCT mode of $\mathrm{HWN}^{*}$, the data session does not have to be dropped even though the congestion in Cell 6 persists. For example, when MT A moves into the congested Cell 6, apart from trying cellular connections, it also associates itself with an RN using either ad hoc frequency or cellular frequency, then the RN may continue transmission with any BS via the multihop relaying structure and the relaying path can be also extended to the area with no cellular coverage. For example, the routing path for an MT in rural place can be even from MT $\rightarrow$ MTs $\rightarrow$ core network; and the corresponding frequencies used can be ad hoc frequency $\rightarrow$ ad hoc frequencies $\rightarrow$ either ad hoc frequency or cellular frequency. In addition, OHAHT of point-to-point ad hoc communications can be another routing mechanism option to further balance traffic load. The simulation results presented in [4] have already proven that inter network traffic management can significantly improve the grade of service, reduce the traffic blocking probability, while maintaining the QoS.

The relay concept extends service range, optimises cell capacity, minimises transmit power, covers shadowed areas, supports inter network load balancing, and supports MANET routing. Theoretically, both the $\mathrm{HWN}^{*}$ system capacity and the transport capacity per MT, when compared to a cellular network, should be improved because the RNs provide relay capability as the substitution of a poor-quality single-hop wireless link with a better-quality link being encouraged whenever possible. Also a higher end-to-end data rate could be obtained if an MT had two simultaneously communicating interfaces.

Using three scaling approaches, we can implement network/simulation dimensioning and estimate how many RNs should be deployed when the number of MTs changes. The three parameters are the number of RNs $m$, the number of MTs $n$, and the system capacity $C$. The asymptotic scaling for the per user throughput as $n$ becomes large is

$$
m \leq \sqrt{\frac{n}{\log n}} .
$$

The per user throughput is of the order $C / \sqrt{n / \log n}$ and can be realised by allowing only ad hoc communications which do not necessarily need RN support, when

$$
\sqrt{\frac{n}{\log n}} \leq m \leq \frac{n}{\log n} .
$$

The order for the per user throughput is $\mathrm{Cm} / \mathrm{n}$, therefore the total additional bandwidth provided by $m$ RNs is effectively shared among $n$ MTs. Finally, when

$$
\frac{n}{\log n} \leq m
$$

the order of the per user throughput is only $C / \log n$ which implies that further investments in relay nodes will not lead to an improvement in throughput and bandwidth optimisation.

\section{RN-PLACEMENT ALGORITHMS}

We explore the relay node placement and $\mathrm{HWN}^{*}$ initialisation problem in this section. The network spectral efficiency was taken by [9] as the objective to optimise RN positioning. The paper made the assumption that the quality 


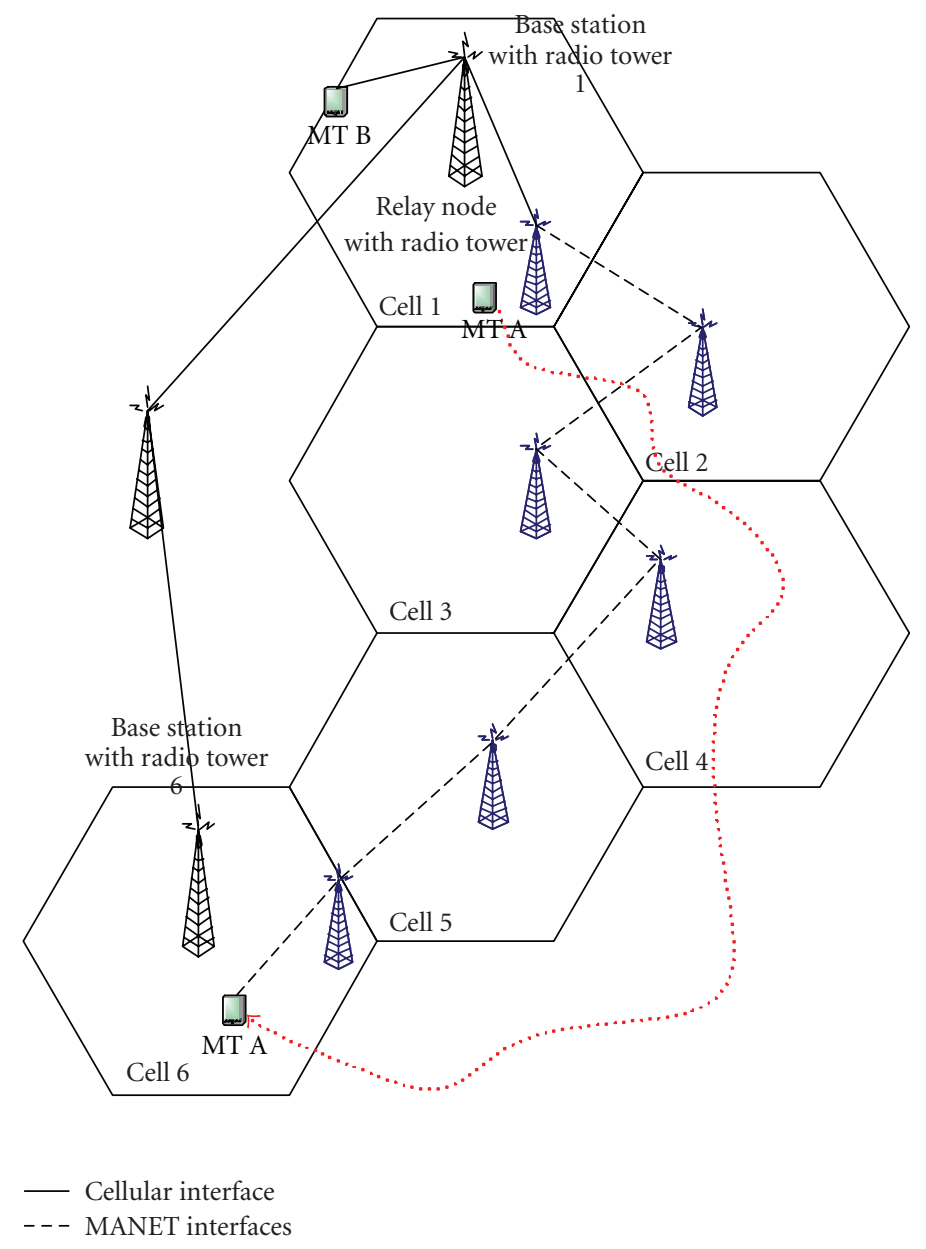

FIGURE 2: Multihop combined transmission example of cellular resource relaying using fixed RNs.

on the links connecting BS $\leftrightarrow \mathrm{RN}$ is always better than the link between RN $\leftrightarrow$ RN. This assumption can be satisfied by establishing line-of-sight (LOS) links between BS and $\mathrm{RN}$ or by designing links that enhance the antenna gains. However, the solution imposes extra difficulty on network planning by complicating transceiver design. In this section, two RN positioning algorithms are proposed, which are packing-based RN placement and heuristic RN placement considering user movement behaviours. The algorithms implementation is to use a minimum number of RNs that enable the relaying of maximum traffic under the media contention from both cellular and ad hoc perspectives.

It is well known from planar geometry that to cover a two-dimensional district with equal-sized circles, the best possible packing solution can be obtained by surrounding each circle by six circles as shown in Figure 3 left. But to have connections between the RNs, an overlap between relay cells is required. We therefore consider a situation where the location of the RNs is centered with maximum coverage. The deployments shown in Figure 3 (left side) are two examples of such pre-engineered approach with a number of RNs in the HWN*. The first deployment tries to cover the entire area while the second one tries to cover densely populated regions.
Heuristic RN placement that we devised has a straightforward design philosophy based on two most important factors, which are user movement behaviour and bandwidth utilisation. By imposing such a plan, we can improve the availability of MTs at disadvantaged locations and enlarge network dimensioning possibility. It is first assumed that RNs can acquire SIR information via local estimation according to the distance. The RN positioning is formulated as a constrained optimisation problem, of which the goal is to maximise the overall network throughput and per node throughput so that majority MTs are better served with guaranteed QoS. The attractor points mobility model deployed on MTs uses macro- and microcontrols to improve user movement experiences, it may be not practical to calculate each MT's trajectory, but probabilities of user reaching a set of frequently visited points can be useful. Coincidentally, the hottest areas are places where most media contention happens, and RN can be located in these points to mitigate the contention. The next step of the heuristic algorithm is to decide the number of RNs needed in solving bandwidth contenting with guaranteed QoS. As shown in Figure 4, after getting traffic load information, the $\mathrm{RN}$ number used for further simulation studies is actually estimated through network dimensioning analysis discussed 


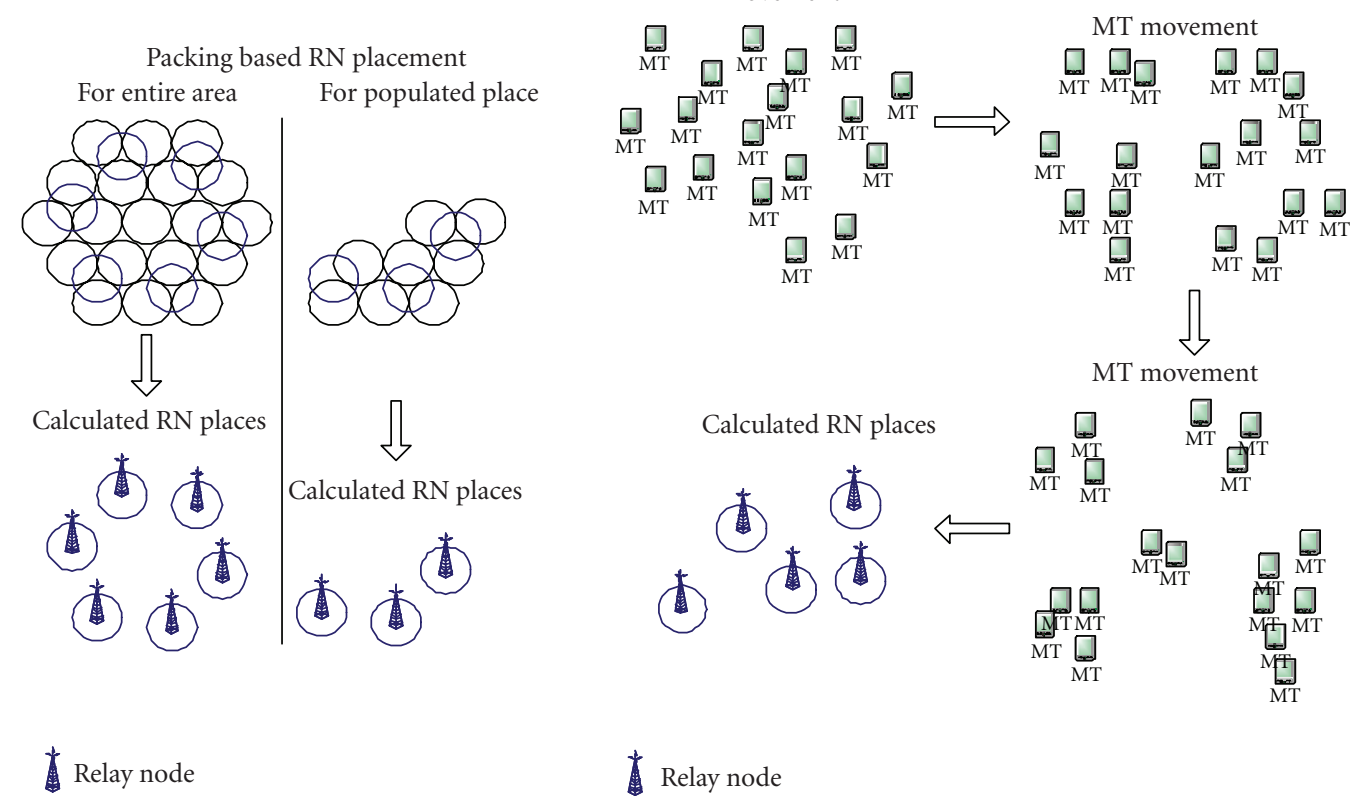

FIgURE 3: Packing-based RN placement and heuristic RN placement.

in Section 2. The migration experiments are carried out to produce a set of candidate points. A hard distant limit $\delta$ is introduced and if distance between one candidate point and any BS is smaller or equal to $\delta$, this point will be eliminated from final list.

The $\mathrm{HWN}^{*}$, after RN placement, is then formed in two stages, which are serving RN, BS association stage, and route identification stage. More details on network formation can be found in [4].

\section{ADAPTIVE DISTRIBUTED CROSS-LAYER ROUTING}

The QoS flows can consume all the bandwidth on certain links, thus creating congestion for, or even starvation of, best effort sessions. Statically, partitioning the link resources can result in low network throughput if the traffic mix changes over time. Thus, a mechanism that dynamically distributes link resources across traffic classes based on the current load conditions in each traffic class is critical for performance. By proposing a cascaded adaptive distributed cross-layer routing (ADCR) for $\mathrm{HWN}^{*}$, we discourage applications from using any route that is heavily loaded with low-priority traffic. Traditional routing strategies that use global state information are not considered. Problems associated with maintaining global state information and the staleness of such information are avoided by having individual MTs infer the network states based on route discovery statistics collected locally, and perform traffic routing using this localised view of the network QoS state. Each application, categorised by service class with the choice of three possible transmission modes, maintains a set of candidate paths to each possible destination and routes flows along these paths. The selection of the candidate paths is a key issue in localised routing and has a considerable impact on how the ADCR performs. The high-priority traffic is given high priority in accessing comparatively expensive cellular resource, while low-priority traffic tries to access low-cost ad hoc resource. Per MT bandwidth is used as the only metric for route local statistics collection since it is one of the most important metrics in QoS routing, furthermore, important metrics such as end-to-end delay, jitter can be expressed as a function of the bandwidth.

We divide traffic sessions into simple service classes which are high-profile users (HPUs), normal-profile users (NPUs), and low-profile users (LPUs). Principally, HPUs get the best QoS, next comes NPUs with smaller medium access opportunities. LPUs are a best-effort class with unused medium resources by other classes. HPUs have the highest access priority in any communication modes of $\mathrm{HWN}^{*}$, and traffic admission of NPUs and LPUs has to consider ongoing HPUs sessions. The NPUs are configured to have a higher probability than LPUs in terms of resource acquisition and this probability is decided by an association level (AL) set. In case of network congestion, CT mode may temporarily become unavailable to NPUs when HPUs are not fully accommodated, while LPUs sessions may be only granted MHCT and OHAHT mode access to mitigate network congestion, reduce transmission delay, and improve per MT throughput. More details of resource acquisition, QoS-based media access control, traffic class coordination, and traffic class association were explained in [4].

The RN has the right to reserve QoS-guaranteed free channels for packet transmission and it maintains a status table that refers to other RNs and it provides information on changing busy conditions or relay failure. The purpose of bandwidth reservation is to let RNs that receive the relaying 


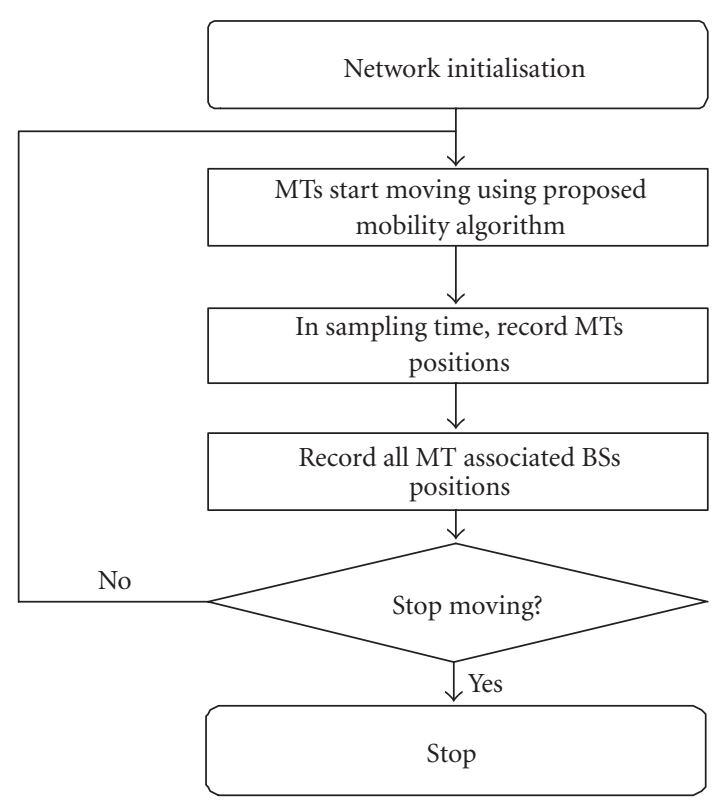

FIgURE 4: Flowchart of heuristic RN placement.

discovery command check if they can provide the bandwidth required for the connection.

To avoid having higher traffic classes being influenced by lower traffic classes in terms of queueing delay, we place a waiting time limitation on each traffic class and force starving packet switch transmission model [4]. A traffic flow maintains two queues: a slot queue and a packet queue, and we decouple slot queue for traffic class identification from packet queue for transmission. Start and finish tags are associated with slots but not packets. When a packet arrives for a flow, it gets added to the packet queue, and a new slot is added to the slot queue. Corresponding start and finish tags are assigned to the new slot. The way to raise priority in slot queue is that the packets related to a high profile have shorter backoff time to increase the probability of early medium access. As for the status table maintenance, information flooding is restricted to a limited scope. Once a positive acknowledgment message is confirmed by requesting $\mathrm{RN}$, the relay paths will not be changed unless resource contention happens. Given the fact that maintaining global RNs channel status in each RN slows down $\mathrm{RN}$ response time, we only require each $\mathrm{RN}$ update neighbouring RNs' information, periodically.

The cascaded ADCR scheme includes three subpacket transmission models, which are the OHAHT, the MHCT, and the CT as illustrated in Figure 5. The communication commands are defined as

(i) ACK/ACCEPT/REJECT/REJHO for the messagedelivery acknowledgment, packet acceptance, packet rejection, and after-rejection handover request.

(ii) SEARCH/SETUP/DATA/BREAK for destination node finding, new connection establishment, packet delivery, and connection teardown. (iii) MOS for MT to choose adaptive transmission mode.

(iv) FAIL used to acknowledge any failure on RN or MT.

(v) LREQ to request a label during the routing, The label is a short, fixed-length identifier. Multiple labels can identify a path or connection from the source MT to the destination MT. The structure of a label message contains flag, flow, cost, traffic class, mobility information, and time tO IIVE (TTL).

(vi) LREP to request a label replay during the label routing in MHCT model.

Time-sensitive multimedia applications have restrictions on end-to-end transmission delay, while FTP data transfers need a minimum guarantee on packet losses. The ADCR should therefore consider differentiated QoS issues while guaranteeing HPUs that agree to pay more than NPUs and LPUs. However, due to the high priority of premium traffic, the global network behaviour as a consequence of this service class, including routing and scheduling of premium packets, may impose significant influences on traffic of other classes. These negative influences, which could degrade the performance of low-priority classes with respect to some important metrics such as the packet loss probability and the packet delay, are often called the interclass effects. To reduce the interclass effects, we proposed in [4] a mechanism based on association level (AL) calculation for load balancing of different service classes. The AL is a set of parameters monitoring channel availabilities, an AL that scores higher than the threshold means that the channels are already occupied by ongoing sessions. The simulation results demonstrated that the proposed mechanism distributes the premium bandwidth requirements more efficiently, and the traffic is better organised and balanced before routing. Figure 5 also presents corresponding process of an MT's association with its serving BS and RN, and simplified ADCR algorithm. As presented in script, if the source MT continues transmitting directly until the SIR falls to a certain level, the traffic re-routing or handovers will be initiated. In rerouting, the model selection priority for HPUs is CT > MHCT > OHAHT, while priorities for NPUs and LPUs are MHCT $>$ CT $>$ OHAHT and OHAHT $>$ MHCT $>$ CT, separately. Also, inter- and intranetwork handover triggers are discussed in paper [4].

\subsection{One-hop ad-hoc transmission}

In OHAHT, the requesting MT first broadcasts SEARCH messages to every node in its transmission range including its associated RN and BS. For example, MT A in Figure 5 broadcasts SEARCH messages, if the destination MT B is within its transmission range and there is no ad hoc-based media contention between MT A and MT B, MT B can respond to MT A with an ACK message. Once MT A confirms the acknowledgment, it starts a connection SETUP session immediately. 

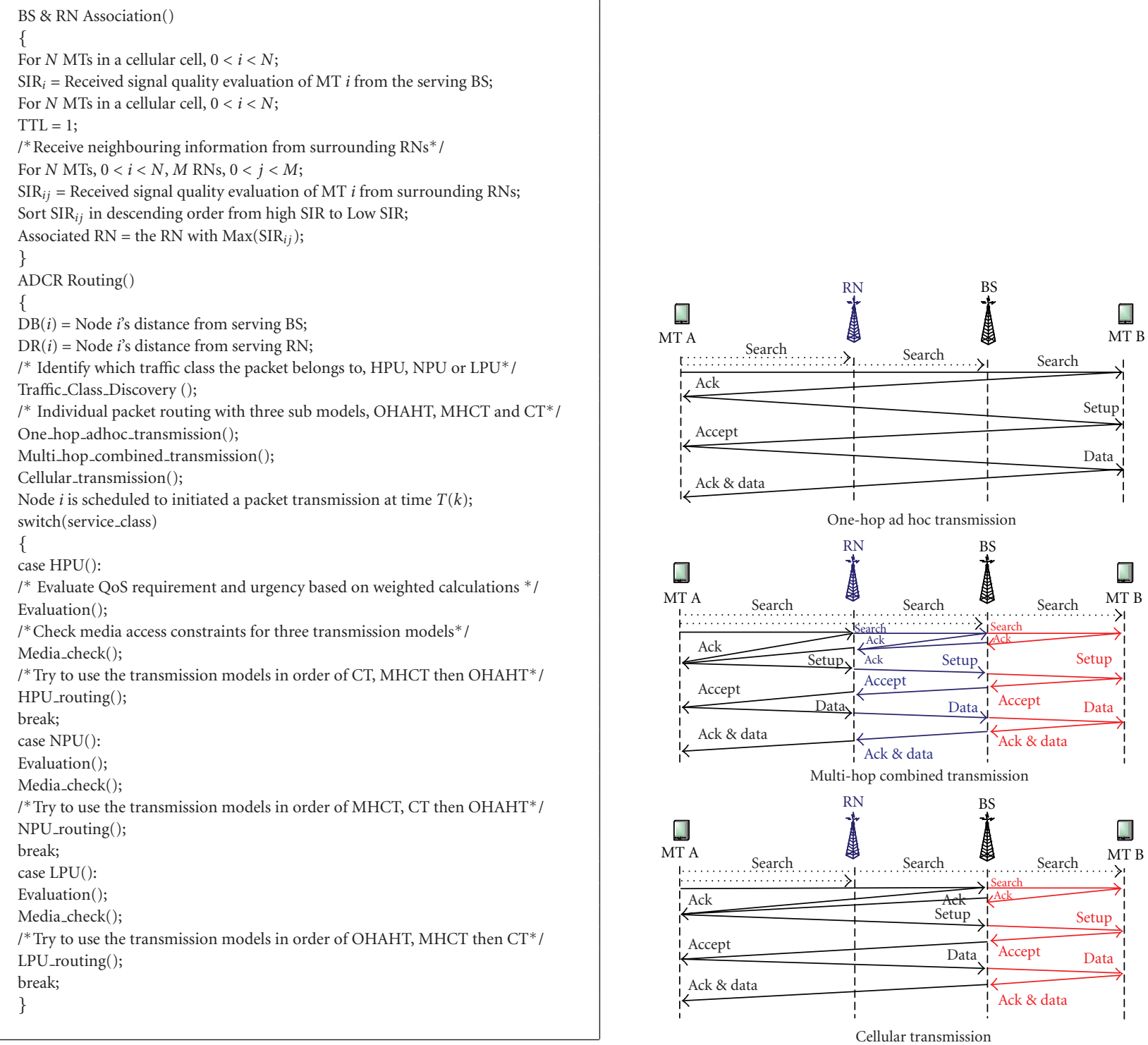

FIGURE 5: Computerised ADCR algorithm and simplified transmission modes illustration.

\subsection{Multihop combined transmission}

The MHCT can involve RNs acting as intermediate nodes for message relaying. Figure 5 shows the connection setup process for communication between MT A and MT B via the RN infrastructure. MT A first broadcasts SEARCH messages to every node to find MT B. After the SEARCH session, MT A may find that the cellular resources can be borrowed through RNs by receiving three ACK messages from the serving BS of MT B, RNs, and the MT B. The positive acknowledgment requires $M T B$ to send an ACK to its serving $B S$, then the serving BS sends an ACK to the RN infrastructure and finally the RNs feedback the ACK to MT A. Once the positive ACK is confirmed, the MT A starts a connection SETUP from $M T A \rightarrow R N$, then $R N \rightarrow B S$, and finally $B S \rightarrow M T B$. The DATA-transmission process follows the same packet delivery route, and further route discovery is prohibited to reduce the signalling overhead.

The label routing concept [10] originated from ATM network is introduced to MHCT mode since RN switching provides faster packet forwarding than routing because its operation is relatively simple. The label switching protocol uses signalling protocol distribute labels and set up new route after the path is computed by the routing module. This requires that the path is pre-established with signalling before it can be used. In reactive MHCT mode with frequent topology changes on both sender and receiver, a high rate of path setup and tear down signaling may occur. It simply can not use separate signalling to set up a new route. Instead, the path finding process dynamically initialised by the LREQ packet carrying a unique label and flow information, where low-path setup delay is guaranteed. The flow information 

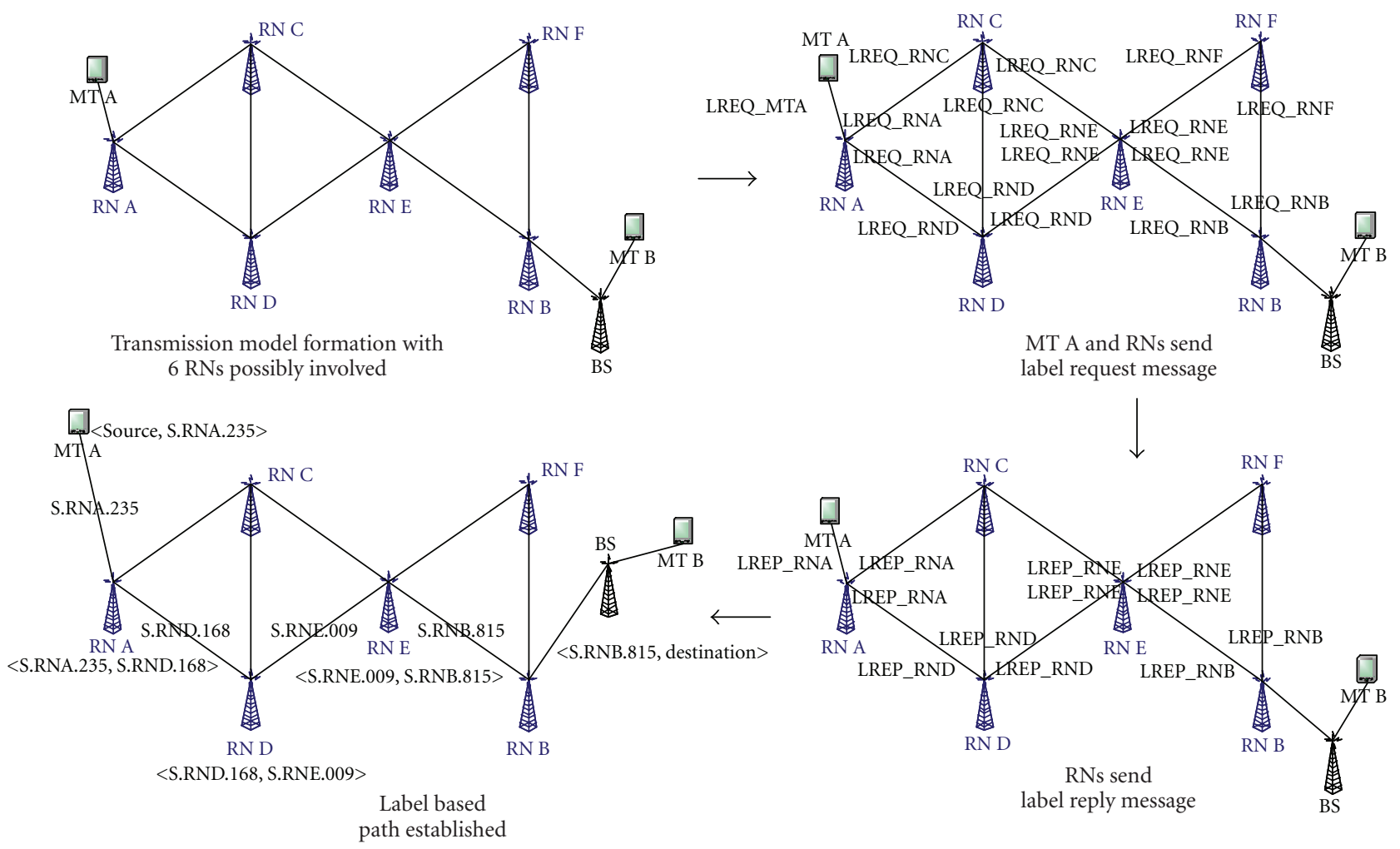

FIgURE 6: Label routing illustration.

contains source address and a flow number is chosen by the source node since the default source address is assumed to be unique.

In finding the destination MT, the source MT creates an LREQ message in which the packet contains IDs, sequence number, and service class of the source MT. This packet also contains traffic flow information, a broadcast ID, and a hop count that is initialised to zero. All RNs that receive this message will increment the hop count. If an RN does not have any information about the destination node, it will record the neighbour's ID where the first copy of LREQ is from and send this LREQ to its neighbours. LREQs from the same node with the same broadcast ID will not be processed more than once. Figure 6 gives an example of label routing in MHCT. In this example, there are eight nodes with duplex connection link.

The MT A first creates an LREQ message and sends it out to its associated RN. Figure 6 illustrates the propagation of LREQ across the RNs and the reverse path at every RN. The reverse path entry is created for the transmission of the reserved label for this path. This label is embedded in the label-reply message LREP. The reserve path entry will be maintained long enough for the LREQ to traverse the path and for RNs to send an LREP to the source MT. Once a path is found in the relay structure, the source MT will check the sequence number (SEQ) of the destination MT in the current path in order to avoid old path information. It should be at least as great as the value entry in the LREQ. Otherwise, the existing path in the table will be discarded. If $S E Q \geq$
$S E Q_{L R E Q}$, it will also check in the current path whether the $\mathrm{QoS}$ requested by the source MT has been satisfied. If not, this request will be discarded. If the source MT still can not find the destination MT B, MT A will increment the hop count in the LREQ by one and then broadcast it to its neighbors. Any duplicated LREQ with same source node ID and same broadcast ID will be discarded. Normally relaybased label routing should have a maximum hop count. However, there is no energy constraints and node mobility issues in our relay infrastructure, thus theoretically any hop count threshold can be possible. We specify the hop count in LREQ as not being larger than 10 as a simulation limitation to avoid computation complexity and if the sender of an LREQ does not receive the reply message, each node only resends the LREQ once for each connection request.

The RN only creates an LREP with the total hop count of this path if hop count, sequence number, and path QoS are all acceptable, the new sequence number of the destination MT is the largest one between $S E Q$ and $S E Q_{L R E Q}$, the best QoS, and a label from its label pool. Then this LREP will be sent back to the source MT along the reverse path entry. The third plot in Figure 6 shows the propagation of the LREP along the reserve paths. Note that both RN C and RN F fail to send the LREP due to hop count, sequence number or QoS issues. The path between the source MT and the destination MT is composed of multiple segments and all data packets are relayed by these segments. Each segment is a real connection between two nodes and labeled by the sending-side node of the LREP in this segment. For example in the the path 
TABLE 2: Characteristics of QoS differentiated users.

\begin{tabular}{llll}
\hline & Low-profile user & Normal-profile user & High-profile user \\
\hline \multirow{2}{*}{ Portion } & Voice 20\% Web 10\% Video 5\% & Voice 15\% Web 8\% Video 10\% & Voice 10\% Web 7\% Video 15\% \\
& Voice dwell/session time: $60 \mathrm{~s} / 120 \mathrm{~s}$ & Web dwell/session time: $120 \mathrm{~s} /$ trace & Video dwell/session time: $120 \mathrm{~s} / 240 \mathrm{~s}$ \\
\hline
\end{tabular}

$M T A \leftrightarrow R N A \leftrightarrow R N D \leftrightarrow R N E \leftrightarrow R N B \leftrightarrow M T B$ showed in the last plot of Figure 6, RNs A, D, E, and B set up the labels of the segments between $\mathrm{A}$ and $\mathrm{D}, \mathrm{D}$ and $\mathrm{E}$, and $\mathrm{E}$ and $\mathrm{E}$, respectively. MT A and RN A, MT B, RN B, and its associated BS are the other two segments. Since the topology of the relay structure is meshed, the source MT can receive more than one LREP. There is a hop count field in the LREP. This field records the total number of hops of the path. The source MT will choose the smallest hop count from the LREPs in the specific limited time. All LREPs that are received after this time threshold will be ignored. And if some available LREPs have the same hop count, the path that has the largest destination sequence number, which means it is the latest path, will be chosen.

The MHCT mode can be also implemented in multihop ad hoc transmissions in copy with rural environment without infrastructure node support. The basic mechanism is almost the same except MT replaces fixed RN and acts as traffic switching nodes. The source MT first tries to establish a connection destination node. If there is no path which can reach the destination node in its local label routing table, or the mobility constrains of MT relaying are violated, the source MT will initiate another path discovery until TTL reaches.

\subsection{Cellular transmission}

The last plot in Figure 5 shows the connection setup of CT model between MT A and MT B via cellular BSs. MT A first broadcasts SEARCH messages to every node to find MT B. After the SEARCH session, the MT A finds that it is able to communicate with MT B directly via BSs, while the connection can be setup through a virtual wireless backbone. The positive acknowledgment of a connection requires MT $B$ to send an ACK to its serving BS, then the serving BS informs the serving BS of MT A or the BS feedbacks the ACK to MT B when both MT A and MT B share the same serving BS. Once the positive ACK is confirmed, MT A starts connection SETUP from $M T A \rightarrow B S$, then $B S \rightarrow B S$, and finally $B S \rightarrow M T B$. The DATA transmission process follows the same packet-switched delivery route. Dynamic channel allocation can be realised in a distributed manner given that the channel usage does not break the two-channel interference constrains [11] which are cosite constraint where there are minimum channel separations within a cell and non-cosite constraint where minimum channel separation between two adjacent BSs is kept.

\section{SIMULATION}

We present various schemes and results of the simulation that have been implemented for the ADCR in this section.
The OMNET++ simulator [12] is used and we generalise all video streaming as real-time services, while web services are referred to as nonreal-time services. Table 2 presents the default QoS profile used consisting of 30\%, $64 \mathrm{Kbps}$ streaming video, $45 \%$ general voice calls, and 25\% nonrealtime web services. The service request portion is distributed and shared among HPUs, NPUs, and LPUs.

The MTs are randomly distributed in 13 regular hexagonal cells ( $1 \mathrm{~km}$ length, $2.6 \mathrm{~km}^{2}$ ) in an $8 \mathrm{~km} \times 8 \mathrm{~km}$ grid. The HWN* attractor point mobility model (HPMM) [4] is implemented. At the simulation start, an MT schedules an $A C K$ message to itself before it determines a new position. After saving the messages, the MT sends a MOVE message to the physical layer and reschedules the $A C K$ to be delivered in a move interval. This metropolitan environment consists of $n$ points which MTs will move towards. The mobility model implementation provides an approach which influences user mobility in a distributed manner with micro mobility, instead of grouping MTs with macro mobility.

BS is placed in the centre of each cell, and from 0 to 1300 MTs are scattered in $\mathrm{HWN}^{*}$. To ensure frequency reuse, 7 frequencies are allocated to each cell with 128 available channels. MT travels from 0 to $80 \mathrm{~km} / \mathrm{h}$ since a relative speed higher than $160 \mathrm{~km} / \mathrm{h}$ is not suitable for the 802.11 radio propagation model, which has limited compensation for channel fading. A node can not continue relaying packet if its speed changes to more than $10 \mathrm{~km} / \mathrm{h}$. The log-normal standard deviation $\sigma$ is set as $10 \mathrm{~dB}$, shadowing correlation distance $\chi_{s}$ is set to $50 \mathrm{~m}$, and the mean $S I R$ value $r_{d}$ is set to $17 \mathrm{~dB}$. Default energy model provided by OMNET++ is implemented, specifically, for a $250 \mathrm{~m}$ transmission range the transmit power used is $0.282 \mathrm{~W}$. Transmit power used for a transmission range of $d$ is proportional to $d^{4}$ [13].

\subsection{HWN $^{*}$ capacity analysis}

The first experiment is to present two pre-engineered RN positioning strategies' influence on the HWN* capacity under various traffic input. The $\mathrm{HWN}^{*}$ network operations are considered, including the process of RN \& BS registration, traffic balancing, routing path discovery, transmission mode selection, and data delivery.

When packing-based RN positioning scheme is implemented in the $\mathrm{HWN}^{*}$, per cell capacity is expected greater than random RN placement $\mathrm{HWN}^{*}$ and normal cellular network under any traffic input. This is because these MTs, which are not serviced in a cell, can use the packed relay path to access other media resources strategically. With the traffic input being increased higher, packing RN-based $\mathrm{HWN}^{*}$ achieves complete connectivity regardless of cellular service penetration percentage. Figure 7 records per cell capacity performance of three scenarios with traffic load 


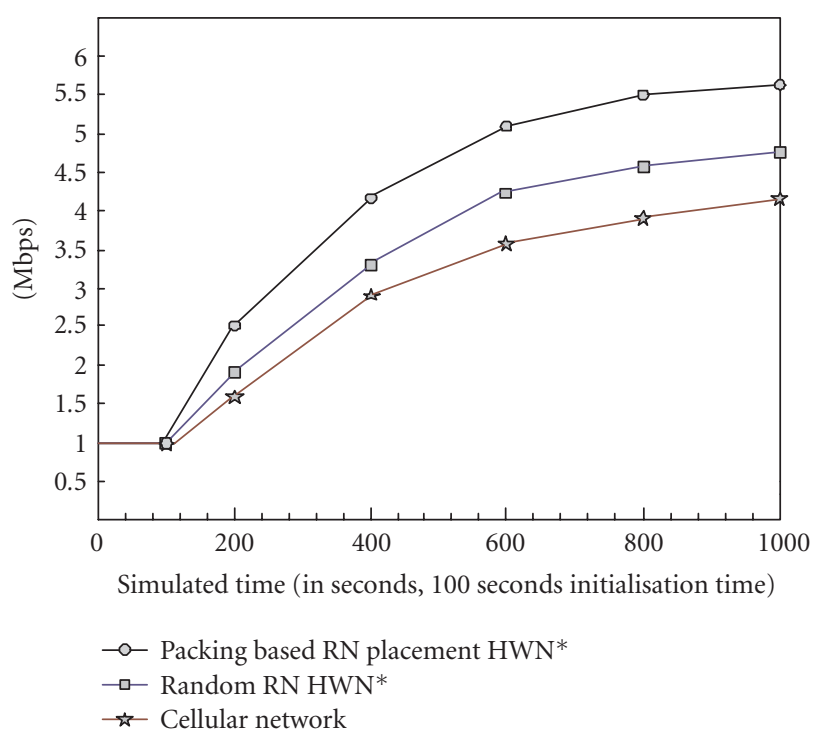

FIgURE 7: Average capacity comparison of packing RN HWN*, random RN HWN*, and cellular network.

being increased. The capacities of both packing RN-based $\mathrm{HWN}^{*}$ and random RN-based $\mathrm{HWN}^{*}$ go up till maximum throughput reaches around $5.6 \mathrm{Mbps}$ and $4.7 \mathrm{Mbps}$, respectively. As we can see from the trend of capacity lines, when the traffic input grows higher, packing RN-based $\mathrm{HWN}^{*}$ outperforms the random $\mathrm{RN} \mathrm{HWN} *$ in terms of network fairness, and its maximum capacity gets close to the theoretical gain with a more uniform communication experience.

Using the same simulation parameters, we also compare per-cell per-second capacity of heuristic placement RN $\mathrm{HWN}^{*}$, random placement RN HWN*, and mobile ad hoc network. The AODV module provided by OMNET++ has been simulated to realise MANET routing. Figure 8 Presents the result. Overall, heuristic RN placement has the highest capacity followed by packing algorithm, random HWN*, cellular network, and MANET (also refer to Figure 7). The extremely low capacity of the MANET is the results of highcontention level, erratic connections, and AODV protocol overhead. Heuristic RN-based $\mathrm{HWN}^{*}$ outperforms packing RN $\mathrm{HWN}^{*}$ under any traffic input, which indicates more traffic is adaptively routed. The maximum capacity of this structure achieves $5.7 \mathrm{Mbps}$.

For packet delivery ratio in the $\mathrm{HWN}^{*}$, the system throughput $(\mathrm{ST})$ is defined as the delivery ratio:

$$
\text { ST }=\frac{\text { Total_number_of_data_received }}{\text { Total_number_of_data_sent }} 100 \% .
$$

In this experiment, we only implement UDP traffic (with no handshaking mechanism) on each MT instead of the default QoS0-based traffic profile, and network operations of the proposed $\mathrm{HWN}^{*}$ are simulated. The packets are sent at constant bit rate (CBR) with a packet size of 1500 bytes and the MTs are added from 0 to 500 gradually as an input parameter to increase the offered load. Figure 9 shows the impact of increased traffic on the packet delivery ratio. It

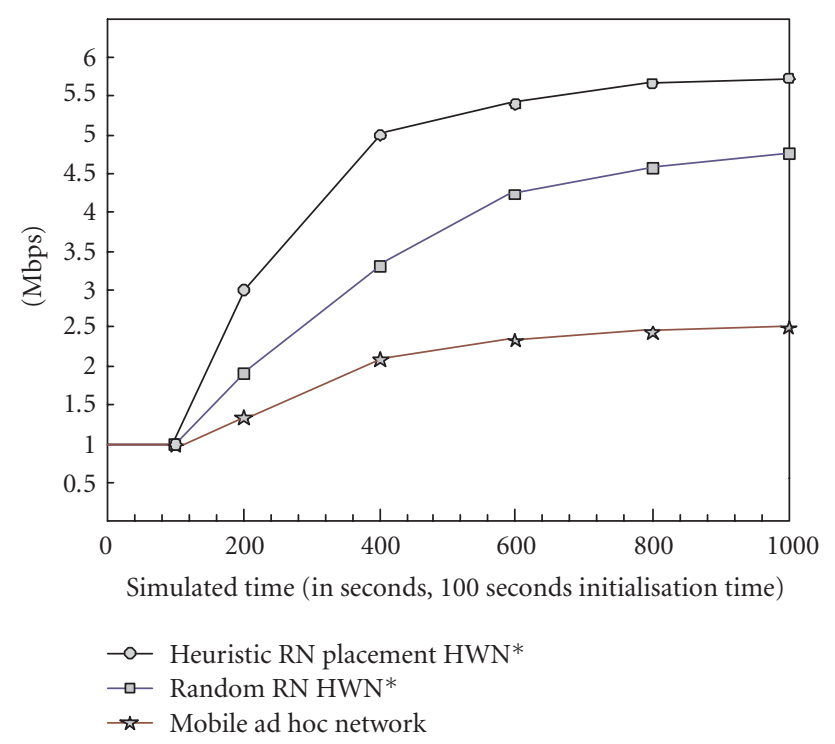

FIGURE 8: Average capacity comparison of heuristic RN HWN*, random RN HWN*, and mobile ad hoc network.

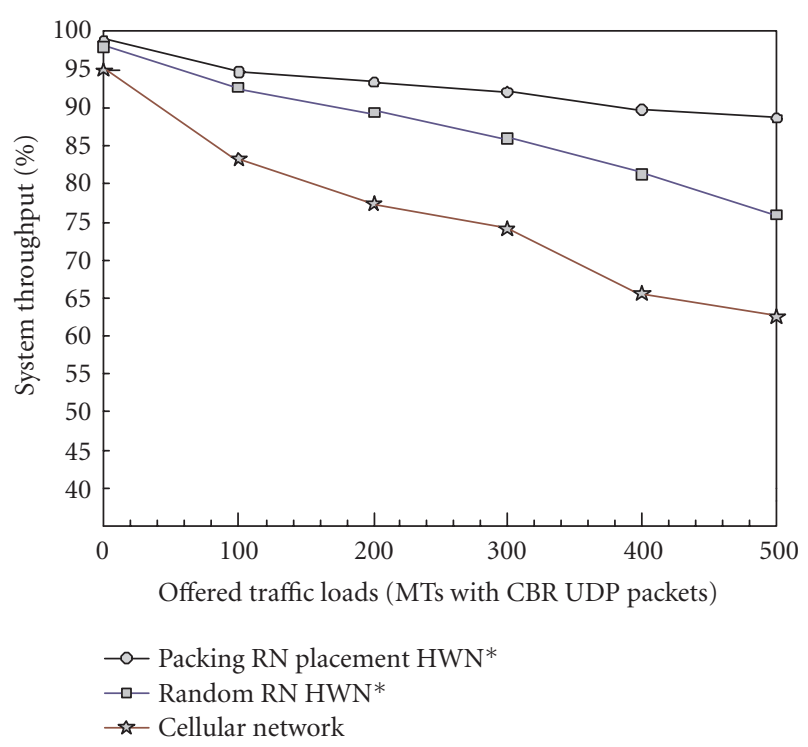

Figure 9: Packing RN HWN*, random RN HWN*, and cellular network throughput versus offered load.

indicates under any traffic input, the ADCR with packing based-RNs placement gives a higher throughput than the $\mathrm{HWN}^{*}$ with random RN placement and pure cellular system. The packet delivery ratio decreases when the UDP traffic load increases, this is mainly due to the congestion. However, packing RN-based $\mathrm{HWN}^{*}$ outperforms random RN HWN* or TDMA network by $12 \%$ and $26 \%$, respectively, when the maximum traffic load is achieved.

In Figure 10, we present the throughput performance for heuristic-based RN placement $\mathrm{HWN}^{*}$ with the ADCR, random RN positioning $\mathrm{HWN}^{*}$ and MANET with the AODV algorithm, respectively. The curve of heuristic RN 


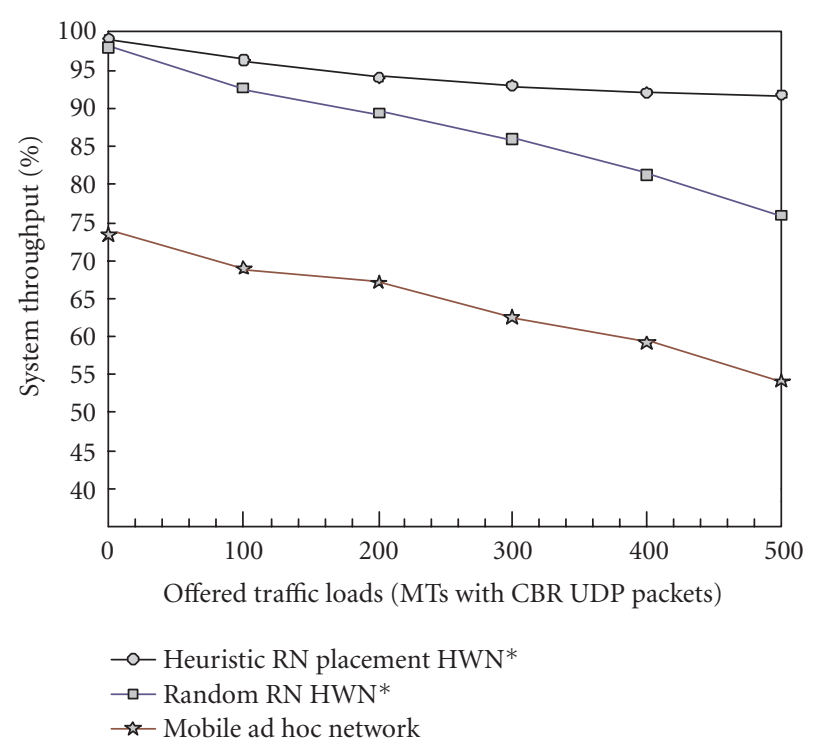

Figure 10: Heuristic RN HWN*, random RN HWN*, and MANET throughput versus offered load.

$\mathrm{HWN}^{*}$ corresponds to a case where all the transmitted packets are maximally received, which can be considered to be an upper throughput bound on this proposed scheme. One can see that the increase of traffic load does not affect too much of the scheme' performance. Overall, heuristic algorithm has the highest throughput followed by packing RN HWN*, random RN HWN*, cellular network, and MANET (also refer to Figure 9). Furthermore, we notice that MANET exhibits a jittering performance with very low throughput under any traffic conditions. When the maximum traffic load achieves, the heuristic-based RN structure outperforms packing-based structure by $3 \%$.

\subsection{Packet transmission delay}

The average packet transmission end-to-end delay of a traffic flow should be directly proportional to the number of hops traversed by the flow, and inversely proportional to the flow's end-to-end throughput, this is an interesting metric to study as the HWN* network itself has a complicated transmission arrangements, which can be seen as hybrid traffic migration of MANET, cellular network, and enhanced packet relay services. The average End-to-end Delay (AED) is defined as

$$
\text { AED }=\frac{\text { Total_number_of_data_received }}{\text { Total_delivery_time }} .
$$

Simplified WINNER and SOPRANO hybrid network infrastructures are therefore simulated with traffic routing functionality. The WINNER concept system realises packet switch through cooperative relaying, and RN operates same resource management functions as cellular BS. In decentralised SOPRANO, route path calculation is exclusively carried out in local MT. A minimum energy routing protocol, as recommended in [14], which maximally saves the

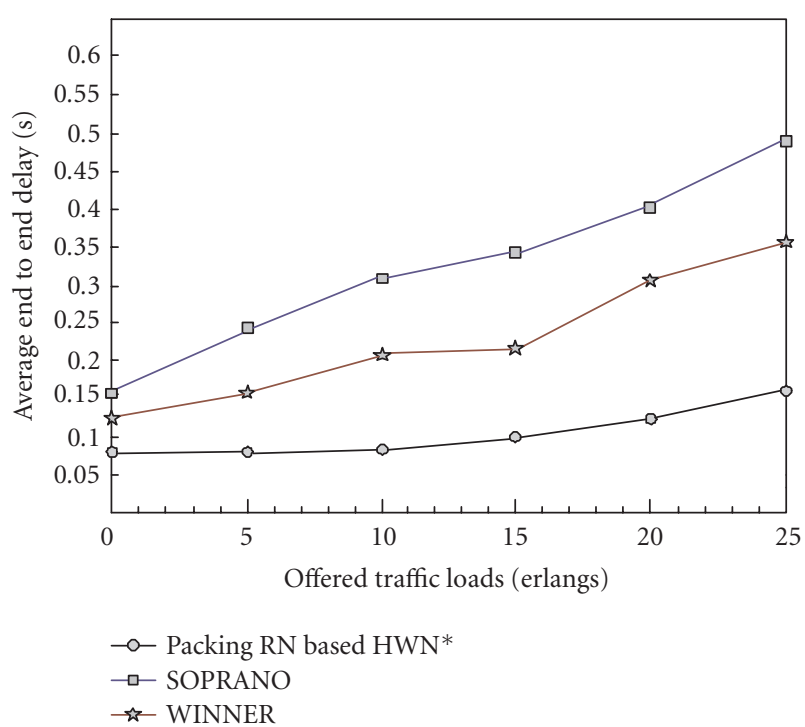

FIgURE 11: Average end-to-end transmission delay of $\mathrm{HWN}^{*}$, WINNER, and SOPRANO.

transmission power, is simulated for SOPRANO. Figure 11 presents the average end-to-end delay versus quantised load offered for three hybrid networks. There is a significant improvement in the delay performance of $\mathrm{HWN}^{*} \mathrm{ADCR}$, compared to the cooperative relaying of the WINNER and the minimum energy routing of the SOPRANO. At 15 Erlangs average offered load, the corresponding average endto-end delays are $0.10,0.21$, and 0.033 seconds and the delay in other systems is almost two or three times larger than the $\mathrm{HWN}^{*}$ ADCR under any traffic load. The ADCR scheme adaptively selects paths with better quality and prevents wasting transmission time.

Figure 12 presents the end-to-end delay comparison result between packing $\mathrm{RN} \mathrm{HWN}^{*}$ with $\mathrm{ADCR}$, heuristic RN HWN* ADCR and heuristic HWN* ADCR + MHCT ad hoc. Interestingly, the MHCT ad hoc mode does not bring too much negative impact on system performance and the position of RNs either does not marginally influent delay performance.

Apart from RN placement plan, the number of relay nodes is another practical parameter to affect the HWN* system performance. The packing-based RN placement HWN* is chosen as the test scenario causes a random RN number to cut off heuristic algorithm that may cause large transmission delay in hot spot. Figure 13 presents delay performance of fully loaded RN, two third RN loaded and one third RN loaded scenarios under increasing traffic load. It clearly indicates that the delay is much less in fully loaded RN plan, compared to the other two scenarios with less infrastructure nodes. One can estimate that an increase of one RN reduces end-to-end delay while improves $\mathrm{HWN}^{*}$ throughput at least $3 \%$ average in a small system domain including seven cellular BSs [15]. However, excessive installation of $\mathrm{RN}$ may not be a preferable approach because a tradeoff exists between management cost and expected system performance. 
TABLE 3: Success route acquire ratio comparison between different user classes.

\begin{tabular}{lcccc}
\hline & HPUs & NPUs & LPUs & Simple HWN* \\
\hline 5 Erlangs/cell & $100.0 \%$ & $100.0 \%$ & $98.0 \%$ & $98.5 \%$ \\
10 Erlangs/cell & $98.1 \%$ & $93.2 \%$ & $91.9 \%$ & $86.2 \%$ \\
15 Erlangs/cell & $97.3 \%$ & $87.0 \%$ & $86.7 \%$ & $77.7 \%$ \\
20 Erlangs/cell & $96.1 \%$ & $84.4 \%$ & $82.5 \%$ & $57.5 \%$ \\
25 Erlangs/cell & $95.0 \%$ & $77.1 \%$ & $72.1 \%$ & $45.1 \%$ \\
\hline
\end{tabular}

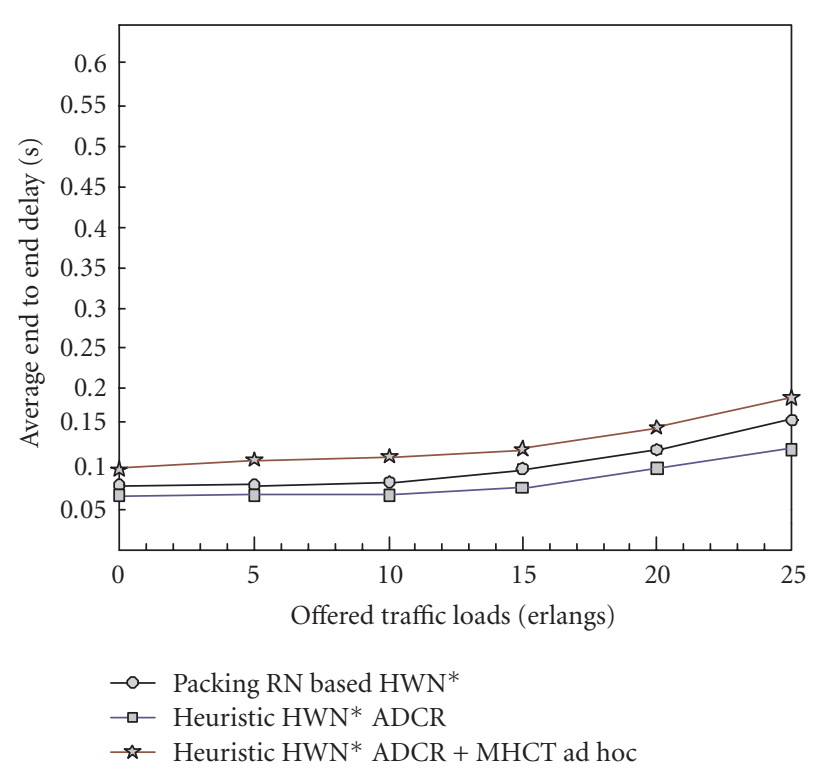

FIgURE 12: Average end-to-end transmission of various $\mathrm{HWN}^{*}$ scenarios.

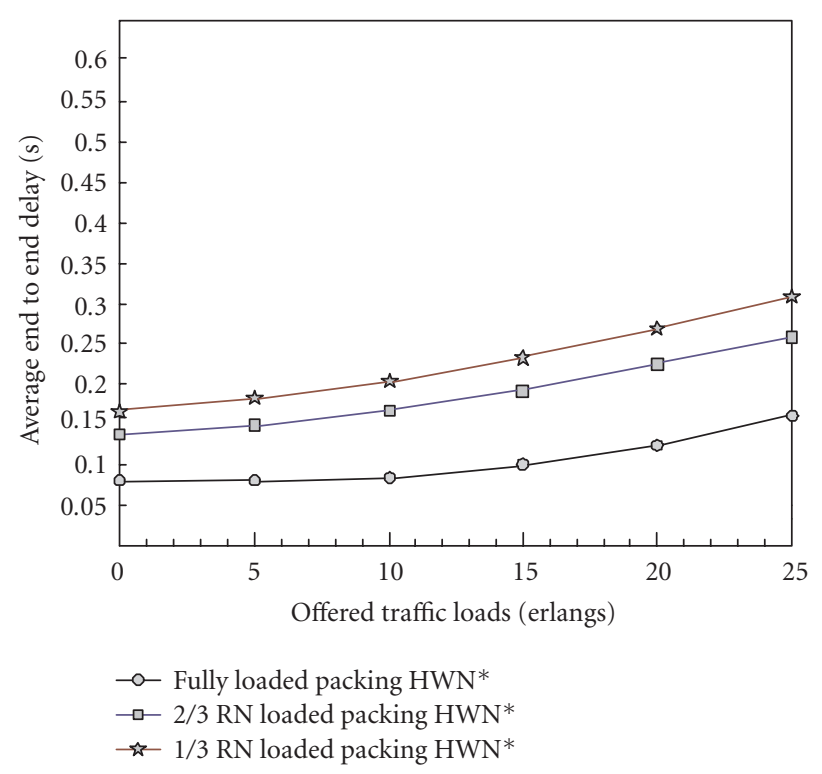

FIgURE 13: Average end-to-end transmission under various RN loads.

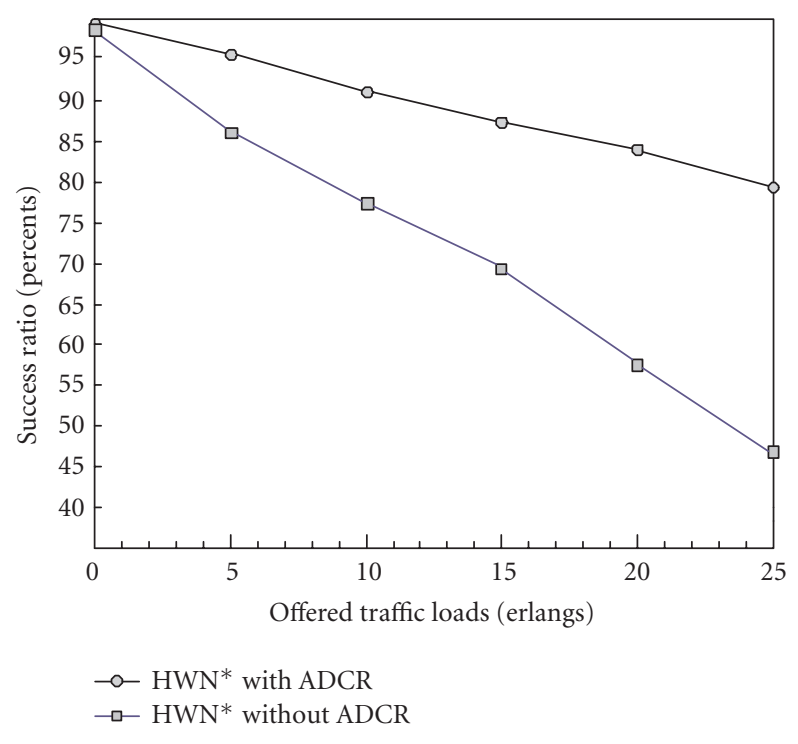

FIGURE 14: Comparisons of success route acquire ratio between $\mathrm{HWN}^{*}$ ADCR and HWN* without ADCR.

\subsection{QoS-based routing analysis}

Experiments are conducted to verify that if the ADCR meets the goal of providing QoS differentiation among different users based on their class profile. To setup a comparison benchmark, we simulated a simple HWN* without any dedicated resource management and routing algorithms. Each packet session in this network has the same privileges when accessing the media resource. The arriving packet sessions are accommodated on a first-come-firstserve basis until all available channels have been occupied. An MT terminates the routing process when it can not find an alternative route. Figure 14 shows the route acquire success ratio with traffic load being constantly increased. The ADCR scheme has the most successful acquire ratio since it always returns a path on-demand and the performance improvement is marginal when the system is heavily loaded. The simple routing algorithm in the $\mathrm{HWN}^{*}$ performs worse than the ADCR due to its limitation on route selection and lack of alternate paths.

Table 3 presents the individual class successful path acquiring probabilities against traffic loads offered for 


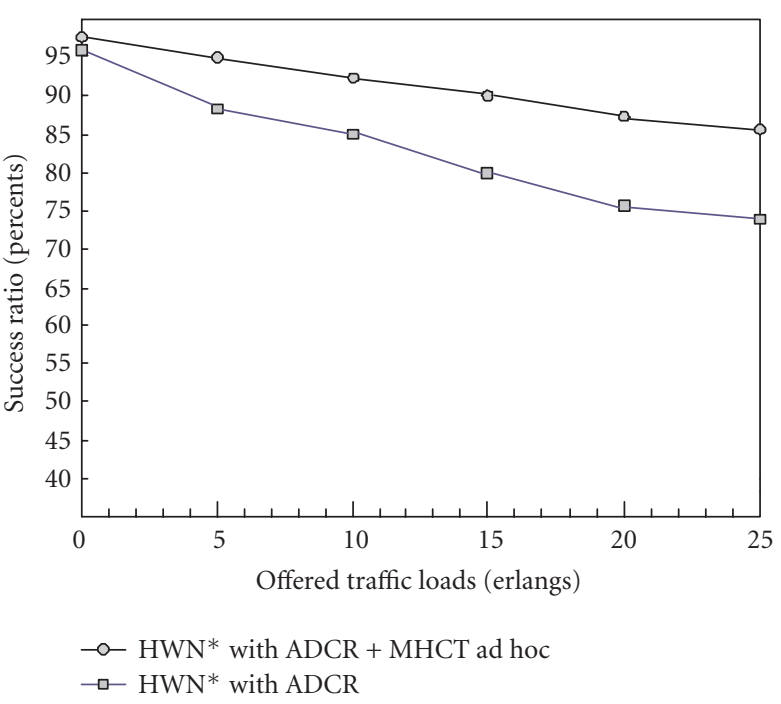

FIGURE 15: Comparisons of success route acquire ratio between $\mathrm{HWN}^{*}$ ADCR and HWN* ADCR + MHCT ad hoc.

different user classes. It can be seen that different results are experienced by user applications in different service classes and for unclassified users in the simple HWN*. Under lowand medium-traffic intensities, the success rates are similar among HPUs, NPUs, and LPUs, since sufficient routes are available and LPUs are not largely affected by HPUs and NPUs communications. However, in the high-traffic intensity case, HPUs and NPUs applications encounter large resource competition in the MAC layer, which consumes a considerable fraction of the radio resource. This may adversely affect the route finding performance of LPUs, in particular when an HPU and NPU traffic hot spot occurs, LPUs are pushed to use the ad hoc communications modes, where the routing process are comparatively unstable compared against the infrastructure-based modes.

An interesting scenario is also investigated where we enable multihop ad hoc communication with MHCT soft relay implementation. When considering route acquire ratio, $15 \%$ of the MTs is configured residing in places outside infrastructure support, while the rest of MTs still migrates using attractor point mobility model. Configuration files for simulation such as user profiles and traffic input are kept the same as the files used in previous QoS-based routing analysis. Figure 15 presents the result comparison between $\mathrm{HWN}^{*}$ with ADCR, and $\mathrm{HWN}^{*}$ with ADCR + MHCT ad hoc. Different levels of route acquire ratio degradation happen in both scenarios. However, under maximum traffic input, the $\mathrm{HWN}^{*}$ with ADCR + MHCT ad hoc system outperforms the HWN* ADCR without multihop ad hoc communication by $11 \%$.

\section{CONCLUSION}

In this article, we have presented an overview of heterogenous wireless networks with the comparison between the $\mathrm{HWN}^{*}$ stage I and $\mathrm{HWN}^{*}$ stage II works. We have devised a cascaded selforganising routing scheme, the ADCR, and enabled the communication in rural places using multihop ad hoc communication. The routing algorithm employs a service class-based approach to discourage applications from using any route that is heavily loaded with low-priority traffic, with three subtransmission modes. Simulation results demonstrate that the ADCR further balances radio resource, reduces transmission delay, and potentially increases the network capacity. The future work will address cross-layer resource selfoptimisation issues in cooperative networks with dedicated relay nodes.

\section{REFERENCES}

[1] R. Beck and H. Panzer, "Strategies for handover and dynamic channel allocation in micro-cellular mobile radio systems," in Proceedings of the IEEE 39th Vehicular Technology Conference (VTC '89), vol. 1, pp. 178-185, San Francisco, Calif, USA, May 1989.

[2] C. Shen, S. Rea, and D. Pesch, "Adaptive cross-layer routing for HWN with dedicated relay station," in Proceedings of the International Conference on Wireless Communications, Networking and Mobile Computing (WiCOM '06), pp. 1-5, Wuhan, China, September 2006.

[3] E. Setton, T. Yoo, X. Zhu, A. Goldsmith, and B. Girod, "Crosslayer design of ad hoc networks for real-time video streaming," IEEE Wireless Communications, vol. 12, no. 4, pp. 59-65, 2005.

[4] C. Shen, S. Rea, and D. Pesch, "HWN* mobility management considering QoS, optimization and cross layer issues," Journal of Communications Software and Systems, vol. 4, no. 3, 2007.

[5] WINNER, "D4.3: identification, definition and assessment of cooperation schemes between RANs," Final deliverable, IST-2003-507581 WINNER, June 2005, https://www.istwinner.org/.

[6] H. Y. Hsieh and R. Sivakumar, "Performance comparison of cellular and multi-hop wireless networks: a quantitative study," in Proceedings of the ACM SIGMETRICS International Conference on Measurement and Modeling of Computer Systems (SIGMETRICS '01), pp. 113-122, Cambridge, Mass, USA, June 2001.

[7] C. Murthy and B. Manoj, Ad Hoc Wireless Networks: Architectures and Protocols, Prentice-Hall, Englewood-Cliffs, NJ, USA, 2004.

[8] B. S. Manoj, K. J. Kumar, C. D. Frank, and C. S. R. Murthy, "On the use of multiple hops in next generation wireless systems," Wireless Networks, vol. 12, no. 2, pp. 199-221, 2006.

[9] H. Hu and K. Yanikomeroglu, "Performance analysis of cellular networks with digital fixed relays," M.S. thesis, Carleton University, Ottawa, Canada, May 2006.

[10] A. Acharya, A. Misra, and S. Bansal, "A label-switching packet forwarding architecture for multi-hop wireless LANs," in Proceedings of the 5th ACM International Workshop on Wireless Mobile Multimedia (WOWMOM '02), pp. 33-40, Atlanta, Ga, USA, September 2002.

[11] C. Shen, D. Pesch, and J. Irvine, "Distributed dynamic channel allocation with fuzzy model selection," in Proceedings of the Information Technology and Telecommunications Conference (ITT '04), Limerick, Ireland, October 2004.

[12] "Omnet++ discrete event simulation system," http://www .omnetpp.org/.

[13] G. L. Stuber, Principles of Mobile Communications, Kluwer Academic Publishers, Norwell, Mass, USA, 1996. 
[14] A. N. Zadeh, B. Jabbari, R. Pickholtz, and B. Vojcic, "Self-organizing packet radio ad hoc networks with overlay (SOPRANO)," IEEE Communications Magazine, vol. 40, no. 6, pp. 149-157, 2002.

[15] C. Shen, D. Pesch, and J. Irvine, "Autonomic TDD link optimising using hybrid wireless network and genetic algorithms," in Proceedings of the 62nd IEEE Vehicular Technology Conference (VTC '05), vol. 1, pp. 262-266, Dallas, Tex, USA, September 2005. 